\title{
Research on fractal characteristics of primary phase morphology in semi-solid A356 alloy
}

\author{
Zheng $L I U^{1,2) *}$, Xiaomei $L I U^{1)}$, Chunhui $H^{2)}$ and Weimin MAO ${ }^{3)}$
}

1) Faculty of Mechanical and Electronic Engineering, Jiangxi University of Science and Technology, Ganzhou 341000, China

2) Jiangxi Nonferrous Metal Processing Engineering Research Center, Ganzhou 341000, China

3) School of Materials Science and Engineering, University of Science and Technology Beijing, Beijing 100083, China

Manuscript received 23 February 2009; in revised form 20 June 2009

\begin{abstract}
Semi-solid slurry of A356 alloy was prepared by low superheat pouring and slightly electromagnetic stirring, and the fractal characteristics of morphology in semi-solid primary phase was researched. The fractal dimensions of primary phase morphology in the semi-solid A356 alloy were calculated by the program written to calculate the fractal dimensions of box-counting in the image of solid phase morphology in semi-solid metal slurry. The results indicated that the morphology of primary phase in semi-solid A356 prepared by low superheat pouring and slightly electromagnetic stirring is characterized by fractal dimension, and the primary phase morphology obtained by the different processing parameters has different fractal dimensions. The morphology of primary phase at the different position of ingot has different fractal dimensions, which reflected the effect of solidified conditions at different positions in the same ingot on the morphology of semi-solid primary phase. Solidification of semi-solid alloy is a course of change in fractal dimension.

KEY WORDS Semi-solid; Morphology of primary phase; Fractal; A356 alloy
\end{abstract}

\section{Introduction}

As a new type of forming technology, semi-solid processing has become one of the most active research fields at the present time. Previous research has indicated that one of the structural characteristics in semi-solid alloy with good forming properties has certain amount of non-dendritic primary phase in the alloy ${ }^{[1]}$. For this reason the deep research on how to obtain non-dendritic primary phase has been carried out. The non-dendritization of morphology in primary phase during preparation of semi-solid slurry reflecting in metallography is a kind of change in irregular images. Morphology of semi-solid structure almost

\footnotetext{
${ }^{*}$ Corresponding author. Professor, PhD; Tel.: +86 797 8312428; Fax: +86 7978312059.

E-mail address: liukk66@163.com (Zheng LIU)
}

DOI: $10.1016 / \mathrm{S} 1006-7191(08) 60118-0$ 
is in complicated geometric shapes, which are irregular or non-smooth, so that it is unable or difficult to be described with Euclidean geometry.

Fractal theory, where the nonlinear courses in nature are looked on as an object of study, is a powerful tool, which probes some rules and physical mechanism implied in unlawful appearance ${ }^{[2]}$. Sun et al. used fractal theory to prove that the structure of solid-liquid interface during directional solidification belongs to the fractal structure which can be quantitatively described by fractal method ${ }^{[3]}$. The grain boundary in $\mathrm{CuAu}$ alloy can be expressed by fractal dimension, but the fractal dimension changes with grain size change ${ }^{[4]}$. The shapes of recrystallized grain boundaries in Ti alloy are self-similar and the fractal dimension of grain boundaries increases with increasing deformation degree and strain rate whereas decreases with increasing deformation temperature ${ }^{[5]}$. Qin and Fan $^{[6]}$ recently introduced fractal dimension $D$ into estimating solid particle structure, in which the fractal dimension $D$ for full spherical particles is 3 . Then, Fan and Chen ${ }^{[7]}$ used experimental results of $\mathrm{Sn}-15 \% \mathrm{~Pb}$ during continuous cooling to estimate value of $D$. The morphology of primary phase in semi-solid A356 alloy prepared by low superheat pouring technology belongs to fractal structure, and there are different fractal dimensions for the morphologies of primary phases prepared by the different technologies ${ }^{[8]}$. These results indicate that the real solidifying structure in alloy possesses the fractal characteristics, in which the theoretical basis is provided for characterizing morphology of structure in solidifying alloy by fractal dimension. It is completely possible that fractal theory can be used to describe and analyze the changing law of structure morphology in solidifying alloy even to the forming mechanism of each kind of composed phases. Therefore, the new inspiration is also derived from the characterizing ability of fractal theory on the irregular morphology, namely the morphology characteristic of semi-solid primary phase can be studied by fractal theory, then the forming mechanism of the semi-solid non-dendritic microstructure can be probed. The primary phases in semi-solid A356 alloy prepared by low superheat pouring and slightly electromagnetic stirring ${ }^{[9]}$ (LSPSES) are taken as examples, and the calculating soft written by Matlab and computing method of fractal dimension in image box-counting are used to study the fractal characteristics of primary phase morphology in semi-solid A356 alloy in this paper.

\section{Fractal Dimension and Calculation of Box-counting Dimension in Images}

Fractal dimension is called Hausdorff dimension, which is one of important characteristics in fractal geometry. It is different from Euclidean dimension of round number in classical geometry but can express some characteristics in images obtained from the nature, which is the main tool describing the complicated structure in fractal body. There are many definitions and computing methods about fractal dimension at present ${ }^{[10]}$. Hausdorff dimension is one kind of the most ancient fractal dimensions used in the manifold "fractal dimension", which possesses the advantage about having definition for any set, but the main disadvantage is difficult to calculate. Therefore, many of the equivalent or approximate dimension definitions are put forward, in which the box-counting dimension is most widely applied. Box-counting dimension is determined by covering through the same shape set, so that it is easier to calculate or estimate. In general, the fractal dimension mentioned refers to box-counting dimension in real application. Box-counting dimension, $D_{\mathrm{B}}$, has a series of the equivalent definitions, but the box-counting dimension used for 
approximate computation can be expressed as follows ${ }^{[2]}$ :

$$
D_{\mathrm{B}}=\lim _{k \rightarrow \infty} \frac{\ln N_{\delta}(F)}{-\ln \delta_{\mathrm{k}}}
$$

where $F$ is a nonempty bounded set in $R^{\mathrm{n}} ; \delta_{\mathrm{k}}$ is the grid side length in cubic body, in which $k$ is generally taken as $1,2,4,8, \cdots, 2^{j} ; N_{\delta}(F)$ is the numbers intersected with $F$.

Graph and image, which include much physical information about objects of study, are carriers calculating fractal dimension. The graph and image can be transformed into digital image after information processing, and two dimension matrix (binary image) expressed by a series of numbers (for example, 0 and 1 ) is obtained finally; then box-counting fractal dimension of digital image can be calculated through the binary image. When calculating the box-counting fractal dimension in the binary image, the calculating method is called pixel-covering method, in which the image stored in computer is thought to be composed of points (pixel) in size of $\delta$.

The specific method of making use of pixel-covering method to calculate box-counting fractal dimension is as follows:

(1) That the image is processed by binarization makes each pixel have black color or white color in the image, so that a data document is obtained, in which the number of rows and columns is corresponding to the numbers of row and column in the binary image. The binary image processed is regarded as a matrix, in which each element is corresponding to the value of pixel.

(2) The data document obtained, namely matrix, is in turn divided into several submatrix, in which the number of rows and columns in submatrix is $k$. The number of non-zero submatrix is written as $N_{k}$, in which $k$ is taken $1,2,4,8, \cdots, 2^{j}$, namely taking the size of $1,2, \cdots, 2^{j}$ pixel as side length to divide the submatrix, thus obtaining the box numbers $N_{1}, N_{2}, N_{4}, \cdots, N_{k}$. Because the size of pixel is equal to image length $L /$ number of a row of pixel in the image, the side length of submatrix, in which the rows and columns are composed of $k$ pixel points, is $\delta_{k}$. The value of $k$ may directly be used to substitute during the specific calculation.

(3) In plane of bi-logarithm coordinate, the data points $\left(\lg \delta_{k}, \lg N_{k}\right)$ are line fitted by least square method, and the negative value $D$ in slope of straight line obtained is the physical box-counting fractal dimension in this image.

\section{Preparing Process and Observation on Solidified Microstructure in Semi- solid A356 Alloy}

The used semi-solid slurry was prepared by low superheat pouring and slightly electromagnetic stirring ${ }^{[9]}$. A356 alloy was melted at $700{ }^{\circ} \mathrm{C}$. As temperature of the melt decreased to the set temperature, the melt was poured into a mould. The mould, a cylinder made of stainless steel with $102 \mathrm{~mm}$ in diameter and $220 \mathrm{~mm}$ in depth, was inserted an electromagnetic stirrer. As the melt was poured into the mould at the set temperature, the stirrer was started and the melt was slightly stirred for short time. Then the mould was quenched in order to maintain the stirred microstructures. The processing parameters were those: the pouring temperature of $630{ }^{\circ} \mathrm{C}$, the stirring time of $8 \mathrm{~s}$, and the stirring powers of $352 \mathrm{~W}$ and $60 \mathrm{~W}$, respectively. 
Some samples are cut from the middle of the ingot prepared, which are polished using standard metallographic practice, etched with $0.5 \%$ aqueous solution of hydroflouric acid. The microstructure of the prepared semi-solid alloy was observed on an optical microscope.

Fig. 1 shows the morphologies of the A356 alloy semi-solid microstructures prepared by LSPSES at $352 \mathrm{~W}$ along the radial direction of the ingot. It is seen from Fig. 1 that the microstructure in the central zone of the ingot basically consists of particle-like primary $\alpha$ phase with smaller size, as shown in Fig.1a. The morphologies of microstructure along the radial direction of the ingot have some changes: from particle-like primary $\alpha$ phase in the central zone changing to rosette-like primary $\alpha$ phase in the transition zone. The microstructure in the transition area mainly consists of rosette-like primary $\alpha$ phase and mixes a small quantity of particle-like primary $\alpha$ phase. The grain size does not much change, as shown in Fig.1b. The change of morphology in the structure continues to the edge zone in the ingot. It is seen from Fig.1c that the microstructure in the edge zone consists of rosette-like primary phase and a few of dendritic-like crystals which are not fully changed into rosette-like primary $\alpha$ phase.

Fig.2 is the morphologies of A356 alloy semi-solid microstructures prepared by LSPSES at $60 \mathrm{~W}$ along the radial direction of the ingot. The "stirring effect" ${ }^{[11]}$ becomes weak due to reduction of the stirring power so that the microstructure in the central zone of the ingot mainly consists of rosette-like and particle-like primary phase with coarser size, as shown in Fig.2a. The microstructure basically consists of rosette-like primary $\alpha$ phase in the transition zone, in which there is more quantity of rosette-like primary $\alpha$ phase, as shown in Fig.2b. The microstructure consists of dendritic-like crystals and a little rosette-

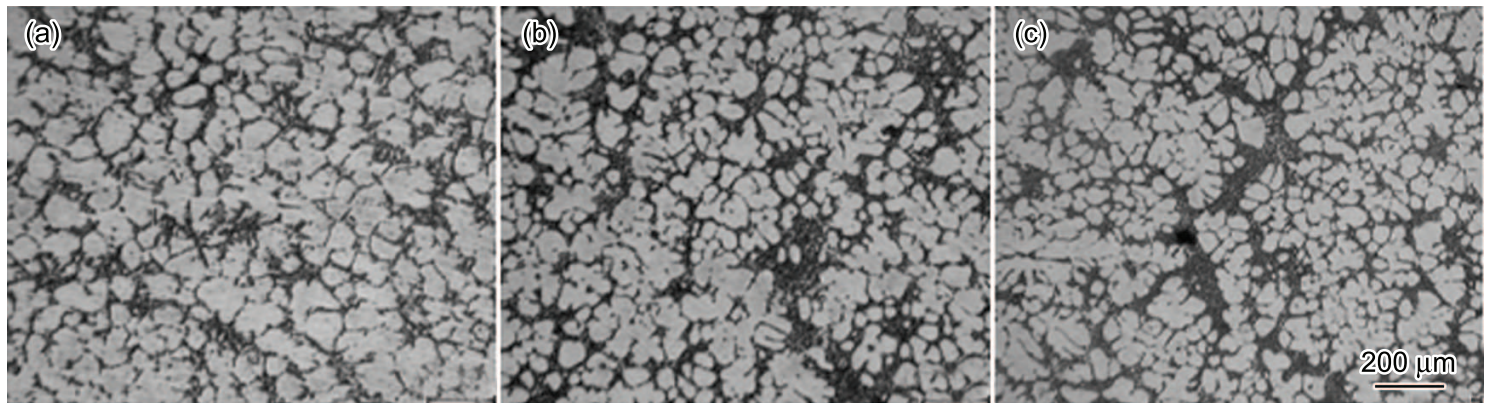

Fig.1 Morphology of A356 alloy semi-solid structure prepared by LSPSES at 352 W: (a) central zone; (b) transition zone; (c) edge zone.

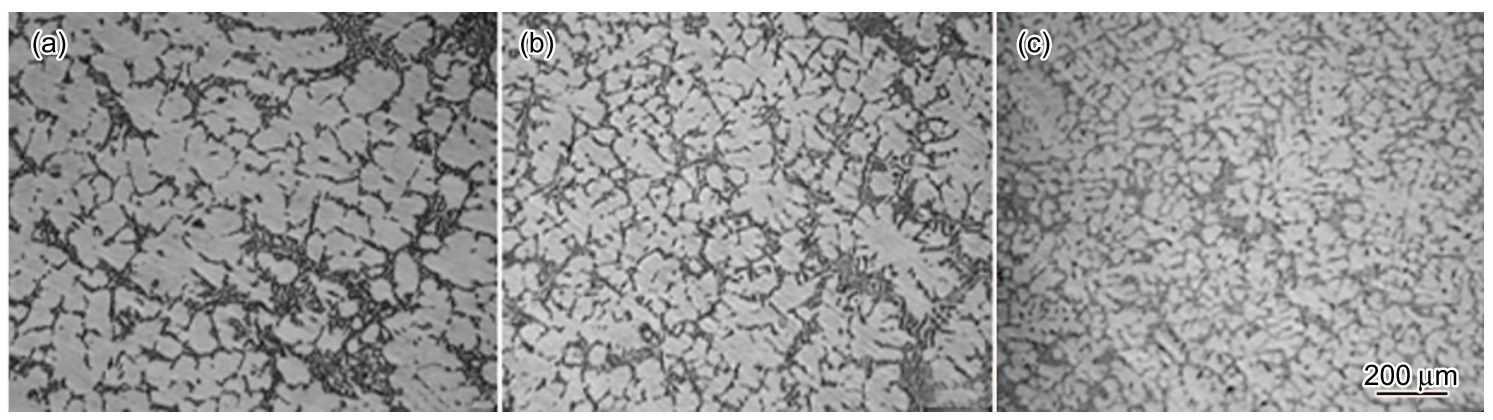

Fig.2 Morphology of A356 alloy semi-solid structure prepared by LSPSES at $60 \mathrm{~W}$ : (a) central zone; (b) transition zone; (c) edge zone. 
like primary $\alpha$ phase in the edge zone, as shown in Fig.2c.

Generally, because of the chilling caused by the mould wall, whether under the condition of stirring or without stirring, there usually is a layer of fine equiaxed grains formed on the ingot surface. During the subsequent solidification, by the effect of direction of heat dissipating on the growth of the crystal, some crystals in the front of the chilling layer will extend to ahead liquid $\mathrm{Al}$ alloy melt at a fast rate to form the main trunk of dendritic crystal, and develop the second arms to form dendritic crystals under the suitable temperature gradient. Once the dendritic is formed, it is difficult to be eliminated, or the dendritic morphology may be changed by the long time powerful stirring ${ }^{[12]}$. In the present research, the morphology of primary phase in the edge of the ingot is obviously improved by low superheat pouring and slightly electromagnetic stirring.

\section{Calculation of Fractal Dimension on Primary Phase Morphology in Semi- solid A356 Alloy}

The fractal dimension of primary phase morphology in the semi-solid A356 Al alloy is calculated by the method provided in this paper, and the fractal characteristic of the primary phase morphology is researched.

First of all, metallographic photos with 50 magnification obtained in the test are processed, then the boundary graph of primary phase is drawn, as shown in Fig.3. The calculating method of box-counting fractal dimension is made use of calculating the fractal dimension, and the results shown in Fig.4 and Fig.5.

It is seen from the results of Fig. 4 and Fig. 5 that the fractal dimensions of primary phase morphology along radial direction in the semi-solid A356 alloy ingot under different stirring power are between 1.60-1.65 and 1.59-1.71, respectively. The differences of fractal dimension reflect the changes of the morphology along the radial direction, in which the changes gradually increase with increasing of the radial distance, and the stronger the stirring power is, the smaller is the difference of fractal dimension in primary phase morphology between the central and the edge zone. Because the value of fractal dimension reflects the complication extent of curve or plane graph to some extent, thus the fractal dimension reflects the complication extent, regular extent of metallographic microstructure

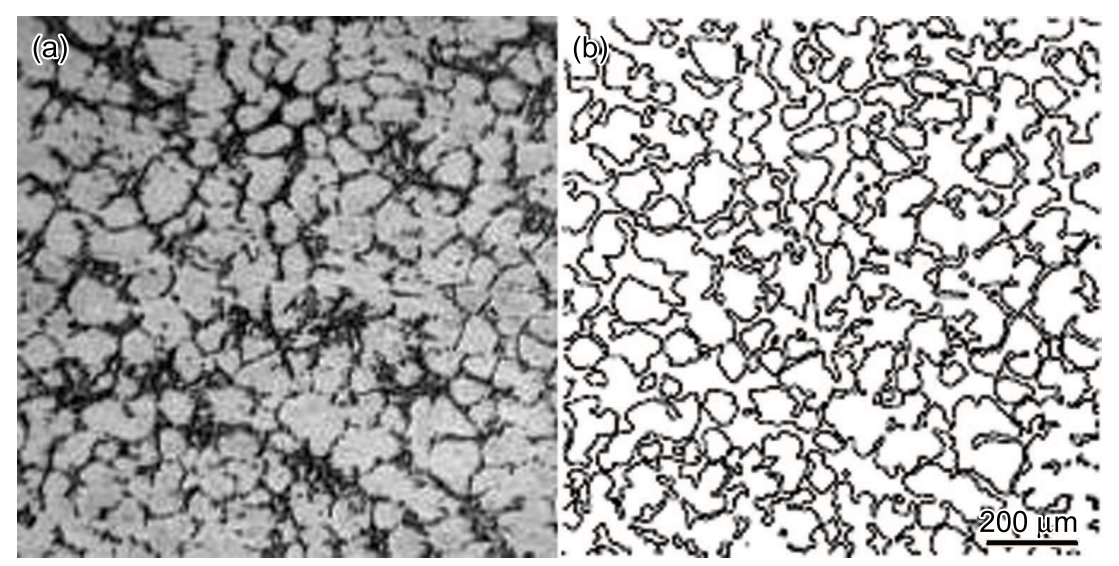

Fig.3 Metallographic photo of the semi-solid A356 alloy and processed boundary graph: (a) morphology of primary phase; (b) processed boundary graph. 

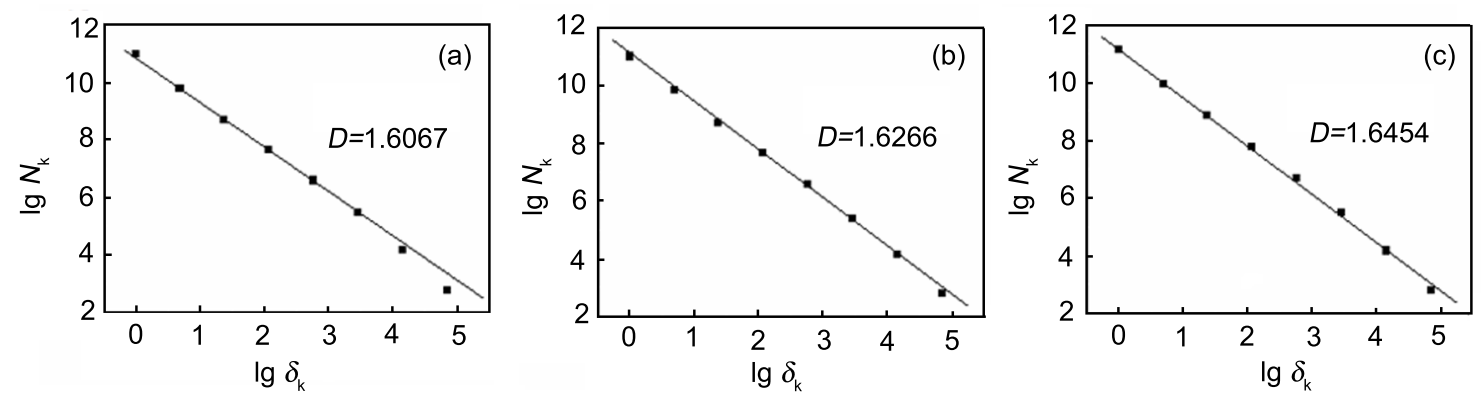

Fig.4 Fractal dimension of morphology of primary phase in the semi-solid A356 alloy stirred at $352 \mathrm{~W}$ : (a) Bi-logarithm graph in the center; (b) Bi-logarithm graph in the transition; (c) Bilogarithm graph in the edge.
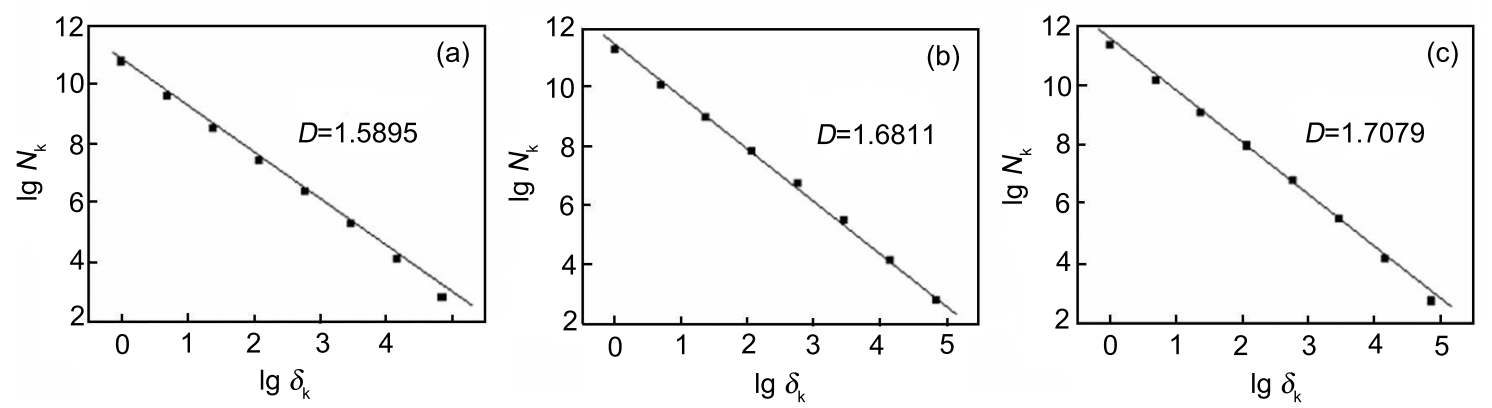

Fig.5 Fractal dimension of morphology of primary phase in the semi-solid A356 alloy stirred at $60 \mathrm{~W}$ : (a) Bi-logarithm graph in the center; (b) Bi-logarithm graph in the transition; (c) Bilogarithm graph in the edge.

and roundness of grains. The smaller fractal dimension expresses that microstructural morphology is simple and regular, and the roundness of grain is better. The little change of the fractal dimension along some direction means that microstructure is uniform. It is consistent with the observation of solidified microstructure in semi-solid A356 alloy.

During formation and growth of semi-solid primary phase, because of the effects of preparing condition and external physical field (such as pouring temperature, stirring power, stirring time, isothermal holding, ultrasonic or mechanical disturbance, addition of modifier and so on), there will be change on the morphology and size structure for the primary phase grain. At the same time, there is other change, including the growth and migration of the grain, even melting and separation of the grain. As seen by the morphology formation of the semi-solid primary phase, the morphology of each semi-solid grain experiences the same change process during evolution, to present the better self-similarity. The fractal dimension of its morphology also correspondingly changes during growth and evolution of the grains, and the fractal dimension also gradually changes with the growth of primary phase grain. The calculation of fractal dimension in semi-solid primary phase indicates that the fractal dimension of dendritic primary phase is greater than that of rosette primary and particle primary phases, which shows that the growing dynamic process of dendritic-like primary phase is more complicated than that of rosette-like primary and particle-like primary phase, so that morphology of semi-solid primary phase obtained is correspondingly complex. 
The value and changing range of fractal dimension in morphology of solidified microstructure of a casting alloy predicts the background information of morphology evolution during growth of constitute phase in the alloy ${ }^{[2,4,8,13-16]}$. It exhibits not only morphology characteristic but also change of solidified conditions. The change of technology conditions during semi-solid processing reflects that morphology of semi-solid primary phase has the different fractal characteristic. There is bigger fractal dimension of primary phase morphology at the edge zone than that at the center and transition zone. Because the edge zone is close to the mould wall, there is a little effect of forced convection caused by electromagnetic stirring on the melt near to the position, to cause the complicated morphology characteristic of primary phase at the edge zone being different from that at the center and transition zone.

\section{Discussion}

It is seen from Fig. 4 and Fig.5 that the fractal dimension $D$ of the primary phase morphology in the semi-solid A356 alloy obtained under the conditions used in this test all is greater than its topological dimension $D_{T}\left(D_{T}=1\right)$. It indicates that the primary phase morphology of the semi-solid A356 alloy prepared at low superheat pouring and slightly electromagnetic stirring belongs to a kind of fractal structure according to the fractal definition presented by Mandelbrot ${ }^{[17]}$. It is known from the present calculations of fractal dimension that all data results are in accord with power law relationship in fractal theory, in which the most data points are near by the straight line with the better linear pertinency. The linear relationship between $\lg N_{k}$ and $\lg \delta_{k}$ in Fig.4 and Fig.5 also further shows that primary phase morphology of semi-solid A356 alloy has the fractal characteristic. It indicates that primary phase morphology of semi-solid A356 alloy prepared at low superheat pouring and slightly electromagnetic stirring has fractal characteristic indeed, and the fractal dimension as a characteristic parameter can be used to describe its primary phase morphology.

Through analyzing the fractal dimension obtained by different stirring power, it is found that fractal dimension of primary phase morphology in semi-solid A356 alloy will change with the change of the preparing conditions, such as stirring power decreasing. This means that solidification of semi-solid alloy is a process of change in fractal dimension.

The fractal structure is the result of the complicated system after evolution, in which randomicity, dissipativity and nonlinearity are the physical mechanism produced fractal structure. There are the numerous complicated factors affecting the morphology during evolution of primary phase morphology in semi-solid A356 alloy, in which they are nonlinear in essence. The physical essence of nonlinearity is dissipativity, but dissipativity is the consequence of randomicity, therefore, the evolution of primary phase morphology in semisolid A356 alloy has fractal character.

\section{Conclusions}

(1) The primary phase morphology of semi-solid A356 alloy prepared at low superheat pouring and slightly electromagnetic stirring can be characterized by fractal dimension.

(2) The primary phase morphology at the different position along the radial direction in the ingot has the different fractal dimension. The change of primary phase morphology 
in semi-solid A356 alloy can be expressed by fractal dimension. The fractal dimension increases with changing morphology of primary phase from particle-like or globular-like to dendritic-like, which reflects effect of solidifying condition at the different position in the same ingot on the morphology of primary phase.

(3) During preparation of semi-solid A356 alloy slurry by low superheat pouring and slightly electromagnetic stirring, the fractal dimension of primary phase morphology will change with the preparing parameters (such as stirring power). This shows that solidification of semi-solid alloy is a process of change in fractal dimension.

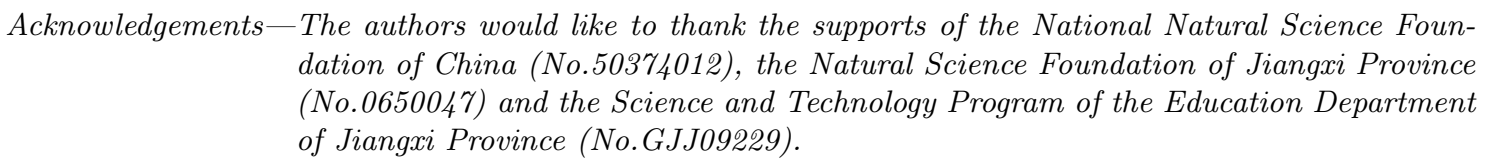
dation of China (No.50374012), the Natural Science Foundation of Jiangxi Province (No.0650047) and the Science and Technology Program of the Education Department of Jiangxi Province (No.GJJ09229).

\section{REFERENCES}

[1] Z. Fan, Int Mater Rev 47 (2002) 49.

[2] J.Z. Zhang, Fractals (Tsinghua University Press, Beijing, 1995) p.30 (in Chinese).

[3] L.L. Sun, L.K. Dong, J.H. Zhang and Z.Q. Hu, J Mater Sci Lett 16 (1997) 505.

[4] K. Hisatsune, Y. Takuma, Y. Tanaka, K. Udoh and K. Kawasaki, J Mater Sci 33 (1998) 4783.

[5] Q. Zhang, P. Li and K.M. Xue, Chin J Nonferrous Met 17 (2007) 1149 (in Chinese).

[6] R.S. Qin and Z. Fan, Mater Sci Technol 17 (2001) 1149.

[7] Z. Fan and J.Y. Chen, Mater Sci Technol 18 (2002) 258.

[8] Z. Liu and F.B. Yu, Chin J Nonferrous Met 19 (2009) 228 (in Chinese).

[9] Z. Liu, W.M. Mao and Z.D. Zhao, Chin Mater Res 20 (2006) 125 (in Chinese).

[10] S.G. Li, Fractals (Higher Education Press, Beijing, 2004) p.44 (in Chinese).

[11] Z. Liu, W.M. Mao and Z.D. Zhao, Trans Nonferrous Met Soc Chin 18 (2008) 573.

[12] K. Sukumaran, B.C. Pai and M.Chakraborty, Mater Sci Eng A369 (2004) 275.

[13] L.L. Sun and L.K. Dong, Acta Metall Sin 29 (1993) 115 (in Chinese).

[14] V.S. Ivanova, I.J. Bunin and V.I. Nosenko, JOM 50 (1998) 52.

[15] V.S. Ivanova, Metal Science and Heat Treatment 43 (2001) 87.

[16] M. Bigerelle and A. Iost, J Mater Sci 41 (2006) 2509.

[17] B.B. Mandelbrot, The Fractal Geometry Nature (W.H. Free-Man, New York, 1982) p.173. 